\title{
Rheological and functional evaluation of the interactions between bitumen and rubber
}

\author{
E.J. Peralta, H.M.R.D. Silva \& J.C. Pais \\ Department of Civil Engineering, University of Minho, Guimarães, Portugal \\ A.V. Machado \\ IPC-Institute of Polymers and Composites, University of Minho, Guimarães, Portugal
}

\begin{abstract}
It is estimated that about ten kilograms of tires are discarded per inhabitant annually. The negative impact of this residue can be reduced, since rubber can be reused as a constituent of asphalt rubber (AR) binder in road pavements. However, the materials which constitute the AR binders and their interaction are not sufficiently characterized. In this work several base bitumens interacted with crumb rubber, in order to produce AR binders, which were subsequently separated, by using a modified "Basket drainage method" to recover the residual bitumen and rubber. EN 12591 or 14023 standards tests and the Dynamic Shear Rheometer (DSR) were used to evaluate the changes in the properties of the binders during AR production. The swelling and depolymerisation changes in the rubber were evaluated through microscopic tests. It was observed that the characteristics of the base bitumen significantly affect the rubber and the AR binder properties (mainly for interactions with softer bitumens).
\end{abstract}

\section{INTRODUCTION}

The introduction of crumb rubber in the production of Asphalt Rubber (AR) mixtures for road pavements should be considered as a sustainable technology which transforms an unwanted residue into a new bituminous mixture highly resistant to fatigue and fracture. However, the addition of rubber into a bituminous mixture increases its complexity. Hence, it is essential to carry out a study to understand the interaction between its constituents, in order to optimize the performance of AR mixtures.

The main objective of this study is to evaluate the properties of the AR binder components (base bitumen and rubber) and their influence in the functional and rheological properties of the AR modified binder. Another objective of this research is to assess the changes that occur in the base bitumen and in the rubber after their reciprocal interaction in the process of the AR binder production. Finally, this work also aims to establish the relationship between the rheological and functional properties of AR binders.

\section{LITERATURE REVIEW ON BITUMEN AND RUBBER INTERACTION}

At present the pavement technology and the evaluation of bituminous mixtures are essentially based on empirical-mechanist studies. Frequently, the materials that constitute asphalt pavements are not sufficiently characterized, more specifically concerning their physicochemical constitution, their rheological properties and their mutual interaction at a microscopic scale.

Therefore, it is essential to develop the knowledge about the interaction between the constituents of bituminous materials used in flexible pavements by carrying out their characterization at a microscopic level, so as to understand their macroscopic structural and functional behaviour. This additional study becomes more significant for modified or unconventional mixtures due to their complexity (e.g. Asphalt Rubber). Actually, beyond the traditional constituents 
of the bituminous layers of the pavements, the use of crumb rubber (recycled from used tires) should be studied as a form of environmental protection and as a pavement performance enhancer.

When crumb rubber is blended at high temperatures with bitumen to produce a modified binder (i.e. wet process), the two materials interact once bitumen components migrate into the rubber causing it to swell (Bahia and Davies, 1994). Initially, the interaction between crumb rubber and bitumen is a non-chemical reaction, where the rubber particles are swollen by the absorption of the aromatic oils of bitumen (Heitzman, 1992).

Absorption of bitumen components by the rubber inevitably depletes the bitumen of the absorbed components and, consequently, modifies its properties, by making it stiffer and more brittle (Singleton et al. 2000, Artamendi et al. 2002, Airey et al. 2003). Furthermore, the rubber particles may also suffer some form of degradation (mainly devulcanization and depolymerisation) when they are mixed with bitumen at high temperatures for prolonged periods of time (Billiter et al. 1997a, Billiter et al. 1997b, Zanzotto et al. 1996). The extent of swelling and degradation depends on the nature of the rubbers, the chemical composition of bitumen and the mixing conditions of time, temperature and degree of agitation. In addition, these processes will determine the mechanical properties of the crumb rubber modified binders (Abdelrahman \& Carpenter, 1999). The asphalt rubber binders are very asphalt dependent: lower saturates and asphaltenes contents improve the asphalt capacity to dissolve rubber (Billiter et al. 1996).

Blending crumb rubber into asphalt is believed to improve its elastic and energy absorption properties, which are directly related to the resistance of the binder to cracking and rutting failures (Gopal et al. 2002). Bahia \& Davis (1995) concluded that the impact of crumb rubber content $(2-20 \%)$ on the reduction of stiffness at low temperature $\left(-20\right.$ to $\left.0^{\circ} \mathrm{C}\right)$ is a linear function of the rubber content and independent of the rubber source and that the lower the stiffness of the asphalt, the less significant the effect of the rubber. The addition of crumb rubber improves the resistance of asphalt binders to low temperature cracking. Optimum crumb rubber contents must be determined for each crumb rubber size and asphalt binder (Gopal et al. 2002).

The use of low penetration grade bitumens in asphalt-rubber mixtures reduces the rate and the amount of swelling of the crumb rubber particles. However, any changes in the rheological properties of the binder following rubber-bitumen interaction could result in the binder becoming embrittled (losing flexibility and capacity to resist cracking and fretting). The use of high penetration grade bitumen will increase the rate and the amount of rubber swelling and therefore the shape and rigidity of the rubber. However, the binder should still have sufficient flexibility following the rubber-bitumen interaction to resist cracking and fretting (Airey et al. 2003). The basket drainage bitumen absorption method was found to be a simple and effective way to monitor rubber-bitumen interaction. The crumb rubber particles absorb bitumen and swell when added together at mixing temperatures. The rate and amount of absorption is mainly dependent on the test temperature and complex chemical nature of bitumen, but only marginally dependent on the bitumen type and grade. In addition to normal oxidation, the residual bitumen experienced further changes in mechanical and rheological properties in terms of increase in stiffness, elasticity, viscosity and reduction in penetration (Rahman, 2004).

Rheology is the science that deals with the deformation and flow of matter. The rheological characteristics of bitumen at a particular temperature are determined by the constitution (chemical composition) and the structure (physical arrangement) of the molecules. Any changes in the constitution, structure or both will result in changes in the rheology. Thus, to understand changes in bitumen rheology, it is essential to understand how the structure and constitution of bitumen interact to influence its rheology (Read \& Whiteoak, 2003).

Asphalt rubber (wet method) is a very special binder if compared to others. Extremely low phase angles at high temperatures/low frequencies and relatively high phase angles and low stiffness at very low temperatures/high frequencies make it a very interesting binder 
(van de Ven \& Jenkins, 2003). The addition of crumb rubber to bitumen decreases the elastic and viscous moduli at low temperatures and, therefore, it causes an increase in binder flexibility. On the contrary, at high temperatures a significant increase in both moduli and a notable drop in the loss tangent values result in a more elastic binder. Furthermore, it can be deduced that the thermal susceptibility of the binder is clearly reduced as a consequence of rubber addition. Consequently, enhanced resistance to permanent deformation, low-temperature and fatigue cracking should be expected in the resulting asphalt rubber mixtures (Navarro et al. 2005).

\section{ASPHALT RUBBER PRODUCTION, SEPARATION AND CHARACTERISATION}

\subsection{Bitumen selection, sample preparation and crumb rubber selection}

A single source of petroleum was used in the experimental work with the aim of reducing the number of factors that can influence the analysis of the results. Four types of bitumens, with penetrations varying between 10 and $200 \mathrm{dmm}$, were collected from the same distillation column, intentionally selected to represent the usual commercial bitumens (pen grades 10/20, 40/50, 60/70 and 150/200). These bitumens were combined in different proportions, as indicated in Table 1, in order to obtain sixteen different samples of bitumen for characterisation.

According to the supplier (RECIPAV), the crumb rubber used in this work was obtained by the cryogenic process and was grinded from $30 \%$ of truck tires and $70 \%$ of car tires. The supplied rubber was sieved in laboratory, in order to obtain and use only the fraction of rubber that went through the sieve ASTM \#20 $(0.850 \mathrm{~mm})$ and retained in the sieve ASTM \#40 $(0.425 \mathrm{~mm})$.

Table 1. Proportions of commercial bitumen used to obtain the sixteen samples of bitumen.

\begin{tabular}{lcccc}
\hline \multirow{2}{*}{ Bitumen } & \multicolumn{4}{c}{ Commercial bitumen used to produce the samples (\%) } \\
\cline { 2 - 5 } Sample & $10 / 20$ & $40 / 50$ & $60 / 70$ & $150 / 200$ \\
\hline A & 100 & 0 & 0 & 0 \\
B & 75 & 25 & 0 & 0 \\
D & 25 & 75 & 0 & 0 \\
E & 0 & 100 & 0 & 0 \\
F & 0 & 75 & 0 & 25 \\
G & 0 & 50 & 0 & 50 \\
H & 0 & 25 & 0 & 75 \\
I & 0 & 0 & 100 & 0 \\
J & 25 & 0 & 75 & 0 \\
K & 50 & 0 & 50 & 0 \\
L & 75 & 0 & 25 & 0 \\
M & 0 & 0 & 0 & 100 \\
N & 0 & 0 & 25 & 75 \\
O & 0 & 0 & 50 & 50 \\
P & 0 & 0 & 75 & 25 \\
\hline
\end{tabular}




\subsection{Production and separation of the asphalt-rubber binder}

The asphalt-rubber binder production facility consisted of an electric heating device in which the aluminium containers with the bitumen were placed. A wire basket (\# $0.420 \mathrm{~mm}$ ) was also placed inside the container in order to allow the final separation between rubber and bitumen constituents (rubber particles were retained in the basket). The procedures for the production of the asphalt rubber binder and for the separation of its constituents were the following:

- Heating of $1 \mathrm{~kg}$ of each base bitumen at $180^{\circ} \mathrm{C}$ and collecting of a sample (BASE);

- Introduction of $17.5 \%$ of crumb rubber (CR) by mass of asphalt rubber;

- Continuous heating of the asphalt rubber binder at $180^{\circ} \mathrm{C}$ for 60 minutes (digestion time) while stirring the binder at a velocity of $230 \mathrm{rot} / \mathrm{min}$ (Fig. 1);

- After finishing the production period, a sample of asphalt rubber (AR) were collected;

- Suspension of the wire basket with the AR binder in an oven at $180^{\circ} \mathrm{C}$ for $15 \mathrm{~min}$, so as to separate its constituents, and collection of a sample of the dry recovered rubber (RR) and of the residual bitumen (RES) (Fig. 2);

- The recovered rubber was washed with toluene for further analysis;

- The residual bitumen was dissolved in toluene and filtered to reclaim some residual rubber particles (FR) eventually suspended in the residual bitumen.

\subsection{Analytical determinations}

The characteristics of the materials involved in the production of AR, crumb rubber and bitumen, were assessed in this work by using several test methods carried out on the samples of binder (BASE, AR, RES) and rubber (CR, RR, FR) collected during the previous procedures.

Following the EN 12591 and 14023 standards, the characteristics of the different samples of binder (BASE, AR, RES) evaluated in this work were the penetration (PEN) at $25^{\circ} \mathrm{C}$

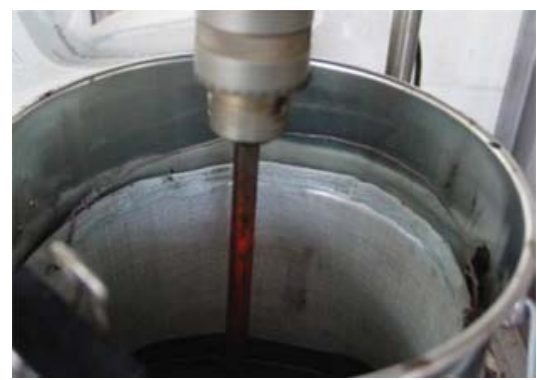

Figure 1. Production of the asphalt-rubber binder.
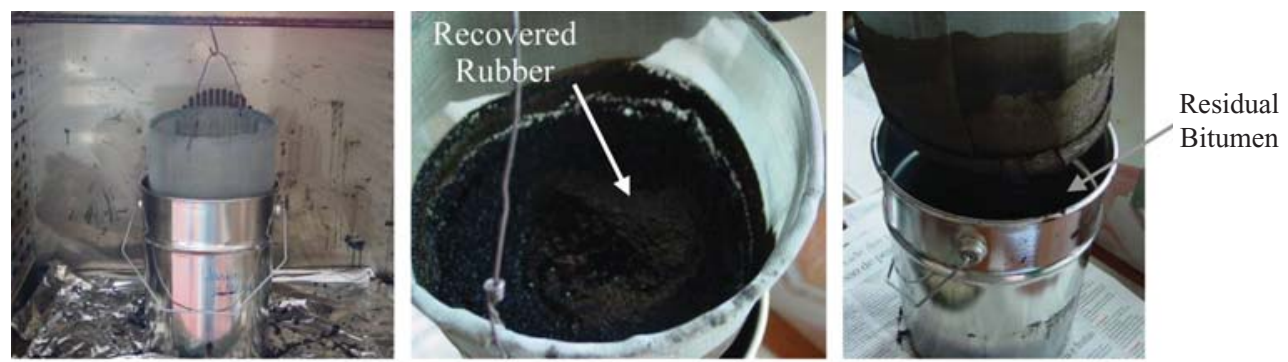

Figure 2. Separation of the AR binder in its constituents (residual bitumen and recovered rubber). 
(following the EN 1426 standard), the softening point, also known as ring and ball (RB) temperature (following the EN 1427 standard) and the dynamic viscosity (DV) at $180^{\circ} \mathrm{C}$, by using a rotating spindle apparatus (following the EN 13302 standard). The elastic recovery (ER) was also assessed in accordance with the ASTM D5329 standard, but only for the AR modified binder. In order to determine the behaviour of all the binders (BASE, AR, RES) at different temperatures (operating, application and mixing temperatures) and frequencies, they were tested in a Dynamic Shear Rheometer (DSR) capable of measuring their rheological properties (in accordance with the EN 14770 standard).

The characteristics of the different samples of rubber (CR, RR and FR) were evaluated by carrying out solubility and microscopic (MIC) tests in the rubber particles before and after the interaction with the bitumen (to study the changes occurred in the production of the AR).

The analytical research performed in this work is systematized in Table 2.

The rheological properties of the binders were measured in a rotational DSR with parallel plate sample geometries of $40 \mathrm{~mm}$ and $1 \mathrm{~mm}$ gap. The rheometer was set up to test in an oscillatory mode to guarantee a dynamic response from the specimen, ensuring that the specimen was tested in the linear region over the temperature $\left(25\right.$ to $\left.180^{\circ} \mathrm{C}\right)$ and frequency range chosen $(0.1$ to $10 \mathrm{~Hz})$. Preliminary tests were carried out at different temperatures and frequencies, in order to select stress values at which the binders are in the linear range. The stress values selected to carry out the DSR tests varied between $1000 \mathrm{~Pa}$ at $25^{\circ} \mathrm{C}$ and $3 \mathrm{~Pa}$ at $180^{\circ} \mathrm{C}$.

The DSR tests began at the lowest selected temperature and frequency and continued to the highest. During the test, the selected oscillatory shear stress was applied to the specimen and the torque $(\mathrm{l})$ and angular rotation $(\theta)$ values were measured, as well as the resulting shear strain.

The final results of the test involved the determination of the standard of the complex shear modulus $\left(\left|G^{*}\right|\right.$ - ratio between peak stress and peak strain), the phase angle $(\delta$ - phase difference between stress and strain), the viscosity and the $G^{*} / \operatorname{sen}(\delta)$ (equivalent to $\left|\mathrm{G}^{*}\right| / \operatorname{sen}(\delta)$ )

Table 2. Analytical determinations carried out in this work.

\begin{tabular}{lllll}
\hline \multirow{4}{*}{ Sample } & \multicolumn{2}{l}{ Analytical determinations } & & \\
\cline { 2 - 5 } Rubber & PEN, RB and DV tests & ER tests & MIC tests & DSR tests \\
A & BASE, AR, RES & AR & RR, FR & BASE, AR, RES \\
B & BASE, AR, RES & AR & & BASE, AR, RES \\
C & BASE, AR, RES & AR & & \\
D & BASE, AR, RES & AR & & \\
E & BASE, AR, RES & AR & RR, FR & BASE, AR, RES \\
F & BASE, AR, RES & AR & & \\
G & BASE, AR, RES & AR & & \\
H & BASE, AR, RES & AR & & \\
I & BASE, AR, RES & AR & RR, FR & BASE, AR, RES \\
J & BASE, AR, RES & AR & & BASE, AR, RES \\
K & BASE, AR, RES & AR & & \\
L & BASE, AR, RES & AR & & \\
M & BASE, AR, RES & AR & RR, FR & BASE, AR, RES \\
N & BASE, AR, RES & AR & & \\
O & BASE, AR, RES & AR & & BASE, AR, RES \\
P & BASE, AR, RES & AR & & BASE, AR, RES \\
\hline
\end{tabular}


values of the binders over a range of test frequencies and test temperatures. Other results of this test presented in this work were $G^{\prime}$ (storage modulus), the real part of the complex shear modulus $\left|G^{*}\right|$ associated with the elastic part of the material behaviour, and $G^{\prime \prime}$ (loss modulus), the imaginary part of the complex shear modulus associated with the viscous part of the material behaviour.

\section{RESULTS AND DISCUSSION}

\subsection{Rubber characteristics and changes observed during the AR production}

Initially, the crumb rubber solubility was assessed according to ASTM D 6814-02 standard, in order to indirectly evaluate the depolymerisation potential of the studied rubber after interacting with bitumen. Two solvents were used in this test, toluene and cyclehexane, being the crumb rubber mass loss equal to $10.9 \%$ and $10.7 \%$ respectively (low depolymerisation potential).

The crumb rubber particles collected during the AR production, before (CR) and after (RR and FR) interacting with different types of bitumen, were cleaned with toluene and analysed by microscopic methods. The results of the microscopic analysis of the rubber that have interacted with the four commercial bitumens used in this work (A, E, I and M) are presented in Figure 3.

The differences between the initial crumb rubber particles and the recovered rubber are evident for all the AR binders produced with the four commercial bitumens. The recovered rubber particles vary in shape and size, whereas the initial crumb rubber particles are clearly more homogeneous. These changes in the crumb rubber morphology cannot result simply from its solubility in the solvent constituents of the bitumen, since the observed changes are visibly superior to $11 \%$ (depolymerisation and devulcanization changes may be occurring).

Comparing the recovered rubber particles from ARs produced with different bitumens, it was possible to observe that the interaction with softer bitumens $(\mathrm{M})$ resulted in a greater variability in the shape and size of the rubber particles than the in harder bitumens (A),

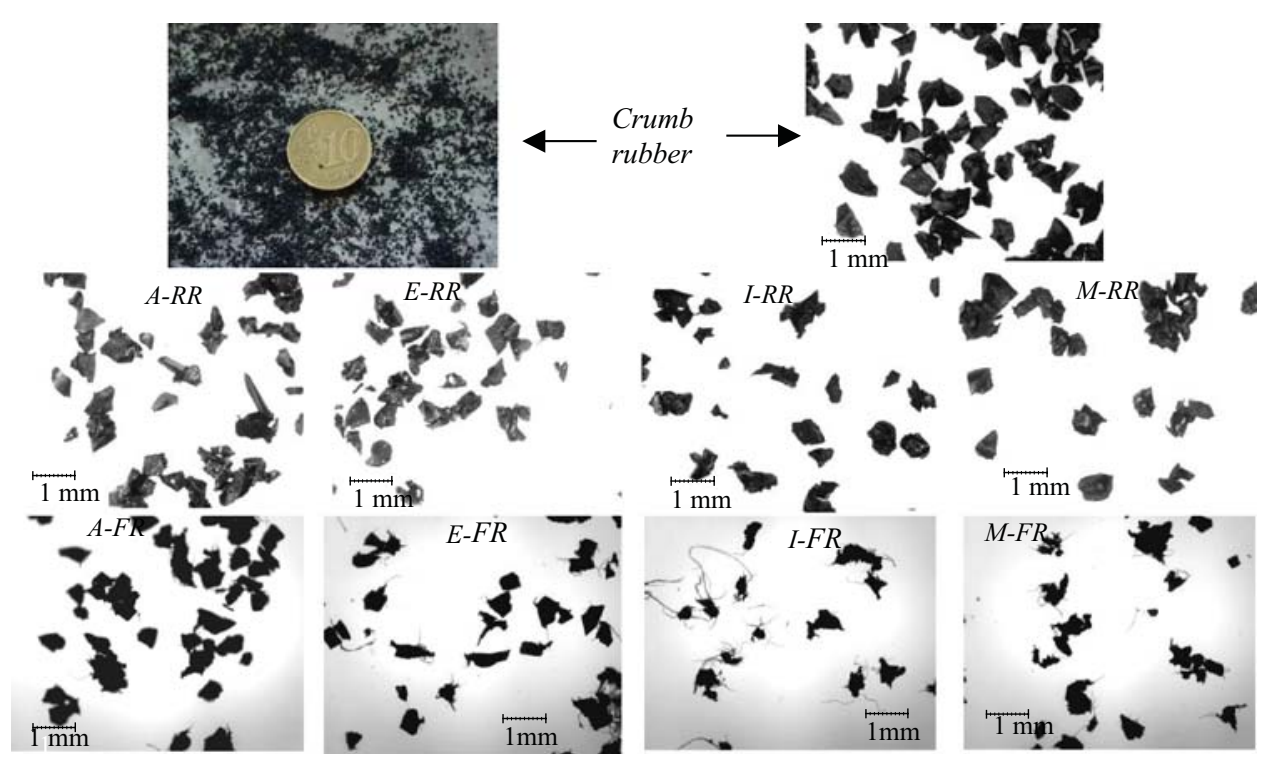

Figure 3. Microscopic photographs of crumb rubber before and after interacting with four base bitumens. 
suggesting that the bitumen constitution has a significant influence in its interaction with the crumb rubber.

The clear evidence of the changes in the rubber particles properties during the AR production can be observed in the microscopic photos of the rubber particles filtered (FR) from the residual bitumen. In fact the size of the crumb rubber particles has inevitably changed during the AR production, since the residual bitumens present some residual rubber particles in its constitution although the initial rubber particles cannot pass through the wire basket.

In conclusion the crumb rubber particles interact with the bitumen in the AR production, and the changes in the shape and size of the rubber particles are more evident in softer bitumens.

\subsection{Functional characterization of the studied binders}

Traditionally, bitumen is the binding material used in flexible pavements. The evolution of technology brought new bituminous materials by using modified binders with more complex behaviour, namely asphalt rubber mixtures modified with crumb rubber.

The functional characteristics of the different samples of binder (base bitumen-BASE; final asphalt rubber binder-AR; residual bitumen removed from the AR-RES) collected throughout the AR production were assessed in this part of the work, in order to evaluate the changes in the binder properties caused by the addition of rubber to the bitumen. Thus, the functional characteristics (penetration, $\mathrm{R} \& \mathrm{~B}$ and Viscosity) of the sixteen (A to P) different base or BASE bitumens used in this study are presented in Figure 4, as well as the properties of the corresponding AR and RES binders. The elastic recovery property is also presented for AR binders.

The results of the different tests were very consistent with the proportions of the commercial bitumen used to produce the sixteen base bitumens. Actually, the changes in the constitution of the samples (from harder to softer bitumens) originated an ordered sequence for the evaluated functional properties, represented by a trend line in Figure 4.

For every functional property evaluated, the AR binder results presented the greatest dispersion, mainly due to the heterogeneity of this material.

As expected, the AR binder presented much lower penetration values and higher R\&B temperatures and dynamic viscosities than the corresponding base bitumen. However, it was noticed that the residual bitumens (RES) had penetration values similar to the AR binders,
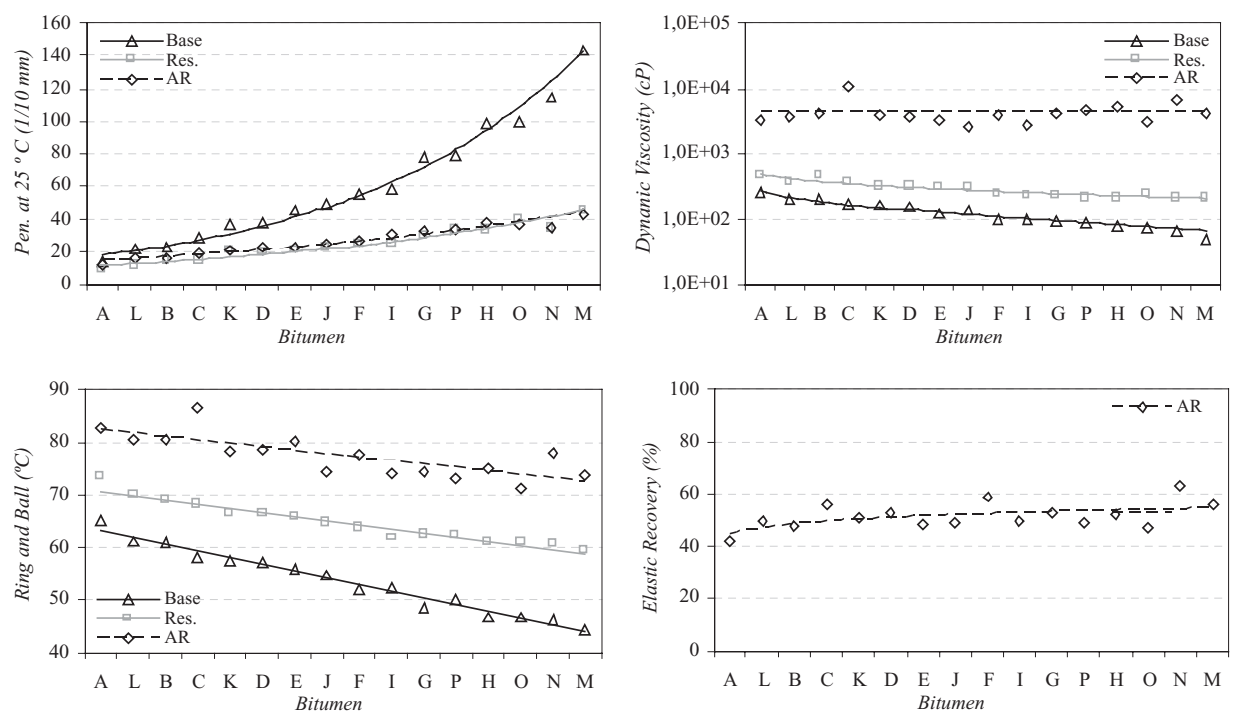

Figure 4. Functional characterization of the different binders (BASE, AR and RES). 
although the $\mathrm{R} \& \mathrm{~B}$ temperature and the dynamic viscosity of these two materials were very different. Thus, the decrease of the AR binder penetration is greatly influenced by the hardening of the base bitumen during the AR production, while the behaviour of the AR binder at higher temperatures ( $R \& B$ and dynamic viscosity temperatures) is more influenced by the crumb rubber particles.

The $R \& B$ test is the most effective to find variations in the behaviour of the different materials, given that it distinguish very well the several base bitumens (A to P) and the different types of binder (BASE, AR, RES). The greatest variation of all the functional characteristics, between the sixteen bitumens, was observed for the base bitumens (BASE). In opposition, the functional characteristics of the AR binders produced with the different bitumens are very similar, mainly the dynamic viscosity and the elastic recovery which hardly vary.

The analysis of the residual bitumen (RES) characteristics demonstrated that significant changes in the base bitumen characteristics are induced by the rubber during the AR production, since the residual bitumen became harder and the remaining functional characteristic were clearly different from the ones of the base bitumen (BASE).

The comparison between the several types of bitumen used to produce AR showed that the highest variations in the functional characteristics of the binder (AR and RES binders compared with the BASE bitumen) are obtained by softer bitumens. This result is consistent with the microscopic evaluation of the most significant changes in the morphology of rubber particles when softer bitumens are used to produce AR binders. Thus, even if the 150/200 bitumen (M) was not generally used to produce asphalt rubber or hot asphalt mixtures, the experimental results showed that it presents the greatest interaction with the crumb rubber, thus being a very good alternative to produce AR binders with final properties similar to those of ARs made with 60/70 bitumens.

\subsection{Rheological characterization of the studied binders}

One of the most significant values normally used to describe the bitumen rheology is $\mathrm{G}^{*} / \operatorname{sen}(\delta)$, specially in the Superpave method developed in the context of SHRP program. Figure 5 shows the isochronal plots of $\mathrm{G}^{*} / \operatorname{sen}(\delta)$ of the BASE bitumen and of the resultant $\mathrm{AR}$ and RES binders at a frequency of $1.0 \mathrm{~Hz}$ and temperatures between 25 and $180^{\circ} \mathrm{C}$ for eight bitumens selected to carry out the rheological tests (A, B, E, I, J, M, O and $\mathrm{P})$. A comparative plot of the three materials (BASE, AR and RES) is also presented for the commercial base bitumens (A, E, I and M).

The isochronal plot of the rheology of the BASE bitumens illustrates the variation of their stiffness and viscoelastic nature with the temperature and type of bitumen evaluated. The lines representing the rheological properties of the different BASE bitumens are in conformity with the previous functional characterisation, i.e. the harder bitumens with lower penetrations and higher $\mathrm{R} \& \mathrm{~B}$ temperature and dynamic viscosity also present the highest values of $\mathrm{G}^{*} / \operatorname{sen}(\delta)$ in the rheology tests for every tested temperature. The change in the gradient of the rheology lines demonstrates a variation in the nature of the bitumen. It is mostly a viscoelastic semisolid material below $100^{\circ} \mathrm{C}$ (when the quick reduction of stiffness results mainly from the loss of the elastic component), becoming a viscous liquid at higher temperatures (when the stiffness decrease is a consequence of the reduction of viscosity). At approximately $180^{\circ} \mathrm{C}$ the bitumen behaviour is almost independent of the bitumen type, trending for a constant and very low value of $\mathrm{G}^{*} / \operatorname{sen}(\delta)$.

The isochronal plot of the rheology of the residual bitumen (RES) is very similar to one of the new bitumen. However, the RES bitumen is stiffer (higher values of $G^{*} / \operatorname{sen}(\delta$ ), mainly below $100^{\circ} \mathrm{C}$ ) and the lines representing the rheology of the different RES bitumens are closer to each other (small differences between the rheology of the several types of RES bitumen, since the interaction with the crumb rubber has hardened the softest bitumens). The inflexion of the rheology curves at $100^{\circ} \mathrm{C}$ is less pronounced than for new bitumens, because the transition between the semisolid and the liquid phases is not so sudden and some elasticity is maintained. 

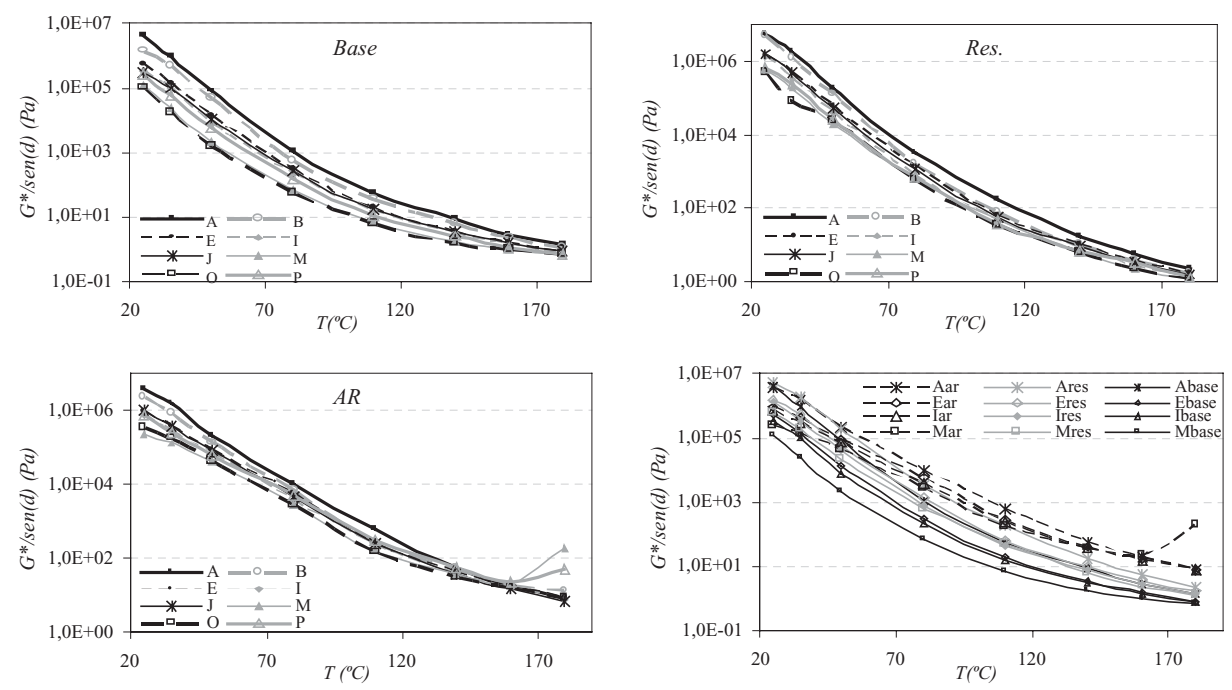

Figure 5. Isochronal plots of $\mathrm{G}^{*} / \mathrm{sen}(\delta)$ rheological characteristic of the studied binders at $1.0 \mathrm{~Hz}$.

The analysis of the rheology of the AR binder showed that it is the stiffest material, being the less susceptible to the change of the base bitumen type (plots are very similar) and to the variation of temperature. In fact, the high increase in the elasticity of the AR binder at all the studied temperatures, caused by the presence of rubber particles, totally changes the rheology of the binder, by reducing the influence of the temperature in the nature of the binder behaviour, mainly for temperatures above $100^{\circ} \mathrm{C}$ (the AR does not totally become a viscous liquid).

Asphalt binders must have a set of characteristics, in order to allow its adequate use in road pavement construction at different temperatures. Binders should not be very rigid at low and medium operating temperatures (to avoid cracking distresses), but they should be sufficiently stiff at high operating temperatures to improve their resistance to permanent deformation. The AR binders fulfil these demands for every base bitumen used, even for the softer bitumen $150 / 200(\mathrm{M})$, since the $\mathrm{G}^{*} / \operatorname{sen}(\delta)$ values at $25-35^{\circ} \mathrm{C}$ are similar to the BASE bitumens, being much higher at $60-80^{\circ} \mathrm{C}$. However, the several AR binders present elevated viscosities at high temperatures, making the mixing and application of AR mixtures difficult.

In pavements, bituminous mixtures performance depends on the weather conditions, as well as on the load and speed of the heavy vehicles. The influence of these parameters in the behaviour of the asphalt binders were evaluated by carrying out rheology tests at different frequencies and temperatures, the results of which are presented in Figure 6 for the commercial bitumen I.

Figure 6 exemplifies, only for one of the tested bitumens (60/70 or I), the typical variation of the rheological behaviour $\left(\mathrm{G}^{*} / \operatorname{sen}(\delta)\right.$ value) of the different types of binder evaluated (BASE bitumen, AR binder and RES binder). The left side illustrates the isochronal plots (for frequencies between 0.1 and $10 \mathrm{~Hz}$ ) of the binders from 25 to $180^{\circ} \mathrm{C}$, while the right side illustrates the isothermal plots (for temperatures between 25 and $180^{\circ} \mathrm{C}$ ) of the same binders from 0.1 to $10 \mathrm{~Hz}$.

At $25-35^{\circ} \mathrm{C}$ the rheology of the different materials (BASE, AR and RES) is less influenced by the frequency than at higher temperatures (superior to $100^{\circ} \mathrm{C}$ ).

The rheological behaviour of the BASE and RES bitumens is similar, especially at higher temperatures (near $180^{\circ} \mathrm{C}$ ), although the RES bitumen has higher values of $\mathrm{G}^{*} / \operatorname{sen}(\delta)$ for all frequencies at $25^{\circ} \mathrm{C}$. The comparison of these two materials with the AR binder showed that, if the temperature increases, the rheology of the BASE and RES bitumens evolves differently from the AR binder (which is clearly stiffer). 

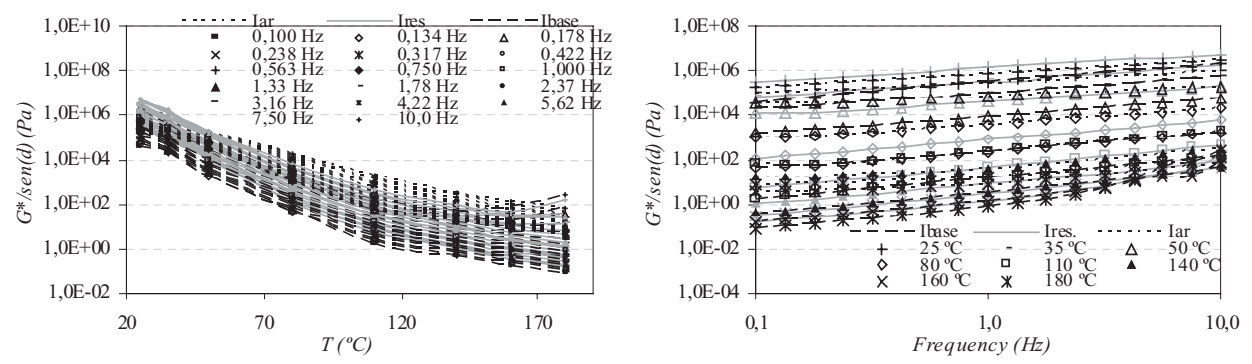

Figure 6. Variation of $\mathrm{G}^{*} / \mathrm{sen}(\delta)$ with temperature and frequency for one binder (BASE, AR and RES).

By observing the isochronal and isothermal plots of the AR binder, it is noticeable that its rheological behaviour is analogous to that of the BASE and RES bitumens for temperatures inferior to $100^{\circ} \mathrm{C}$ at all frequencies. However, for temperatures above $100^{\circ} \mathrm{C}$ the behaviour of the AR binder almost becomes independent of the frequency, trending for a single value of $G^{*} / \operatorname{sen}(\delta)$ clearly superior to the ones of the BASE and RES bitumens (except for frequencies near $10 \mathrm{~Hz}$ ).

Finally, by analysing the effect of the frequency in the rheology of the binders for the different isothermal curves, it is possible to observe that all curves have the same shape (being almost parallel), for all the materials, for temperatures below $100^{\circ} \mathrm{C}$, presenting higher values of $G^{*} / \operatorname{sen}(\delta)$ as the temperature reduces and the frequency increases. The influence of the frequency is still the same for the AR binder at higher temperatures and frequencies, but for the BASE and RES bitumens the influence of the frequency in the rheology increases substantially for temperatures above $150^{\circ} \mathrm{C}$ and frequencies superior to $3.0 \mathrm{~Hz}$.

\subsection{Comparison between the rheological and functional properties of binders}

During the pavement life cycle, the asphalt binders used in bituminous materials should always present the adequate characteristics, initially during the mixing and application phases at elevated temperatures (Fig. 7), then during the operating phase, in order to resist to the weather and traffic actions at low and medium temperatures (Figs 8,9) and, finally, in the removal and recycling of the distressed mixtures (this last phase was not studied in this work).

The results of the two different approaches used to assess the binder behaviour (functional and rheological for a reference frequency of $1 \mathrm{~Hz}$ ) will be compared next for the eight BASE, AR and RES binders (A, B, E, I, J, M, O and P) evaluated in this study.

At mixing and application temperatures, the functional parameter measured in this work was the dynamic viscosity at $180^{\circ} \mathrm{C}$. Figure 7 compares the dynamic viscosity at $180^{\circ} \mathrm{C}$ with two rheological properties measured at $180^{\circ} \mathrm{C}$, the complex viscosity $\left(\eta^{*}\right)$ and the complex shear modulus $\left(\mathrm{G}^{*}\right)$. The values of $\mathrm{G}^{*}$ are approximately the same of $\mathrm{G}^{*} / \operatorname{sen}(\delta)$ at this temperature $\left(180^{\circ} \mathrm{C}\right)$, because the phase angle $(\delta)$ is nearly $90^{\circ}$. In fact, at this temperature the binders behave as a viscous liquid with an insignificant elastic component.

The relations between the functional and rheological properties at $180^{\circ} \mathrm{C}$ represented in the graphics of Figure 7 are nearly linear, with a similar positive slope for the BASE, AR and RES binders. The AR binder is clearly more viscous than the BASE and RES bitumens, being the viscosity of the AR binder less dependent on the base bitumen characteristics. The comparison between the dynamic viscosity (functional) and the complex viscosity (rheology) presented the best relation, being the slope nearly equal to 1 (since $1 \mathrm{~Pa} . \mathrm{s}=1000 \mathrm{cP}$ ).

At high operating temperatures causing rutting distresses, the functional parameter measured in this work was the $\mathrm{R} \& \mathrm{~B}$ softening temperature. Figure 8 compares the $\mathrm{R} \& \mathrm{~B}$ temperature with two rheological properties measured at 50 and $80^{\circ} \mathrm{C}$, the $\eta^{*}$ and the shear modulus $\left(\mathrm{G}^{*}\right)$.

The set of lines presented in Figure 8 show an exponential relation between the R\&B functional properties of all the binders and the corresponding rheological parameters $\left(\eta^{*}\right.$ and $\left.G^{*}\right)$ 

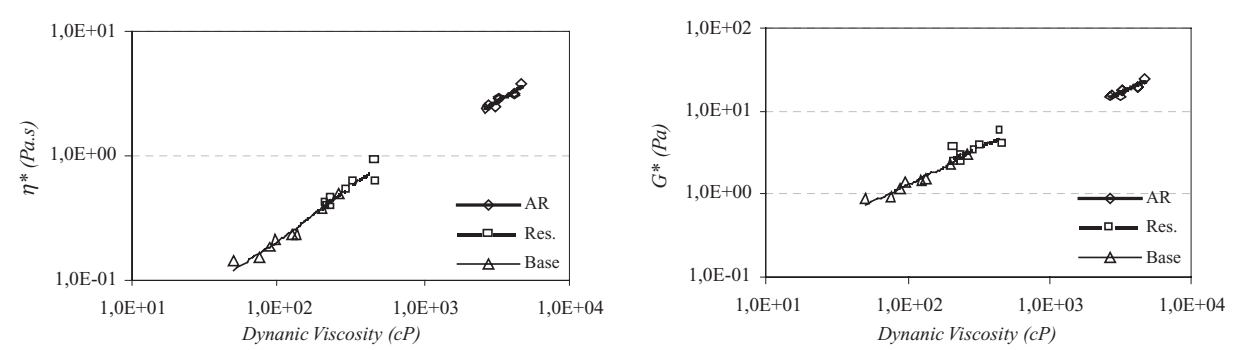

Figure 7. Rheological vs. functional properties at production and application temperatures.
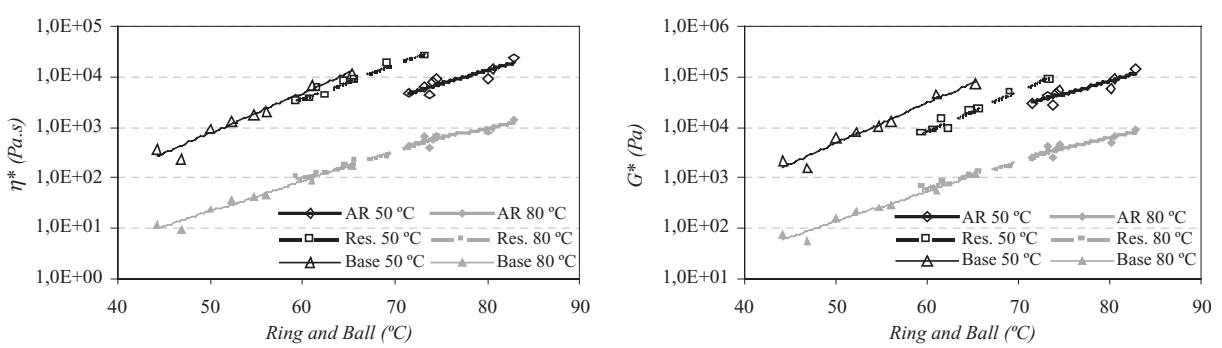

Figure 8. Rheological vs. functional properties at high operating temperatures cause rutting.
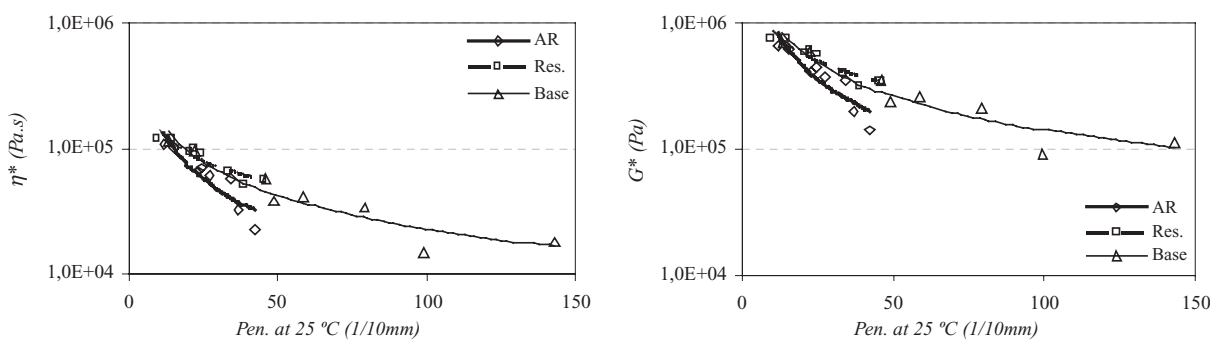

Figure 9. Rheological vs. functional properties at operating temperatures cause fatigue cracking.

measured at $50^{\circ} \mathrm{C}$ and $80^{\circ} \mathrm{C}$. The range of values of the $\mathrm{R} \& \mathrm{~B}$ results is visibly inferior to the rheological classification, and thus the rheology axis is presented in a logarithmic scale.

At $50^{\circ} \mathrm{C}$ the differences between the different binders (BASE, AR and RES) are clear, since the lines relating the functional and rheological properties of those binders are visibly disconnected. This occurs because the softening temperature of several binders is superior to $50^{\circ} \mathrm{C}$, mainly the $\mathrm{AR}$ binders that only melt above $70^{\circ} \mathrm{C}$. At $80^{\circ} \mathrm{C}$ the relation between the functional and rheological properties can be drawn by a continuous line adjusted to all binders. Thus, the R\&B temperature has a very good correspondence to the rheological properties at $80^{\circ} \mathrm{C}$. Figure 8 also confirms the previous observations that the softer AR binder is stiffer than all the BASE bitumens, especially if this comparison is made at $80^{\circ} \mathrm{C}$.

At medium operating temperatures causing fatigue cracking distresses, the functional parameter measured in this work was penetration at $25^{\circ} \mathrm{C}$. Thus, Figure 9 relates the penetration value of the several binders to two rheological properties measured at $25^{\circ} \mathrm{C}$, namely the complex viscosity $\left(\eta^{*}\right)$ and the complex shear modulus $\left(\mathrm{G}^{*}\right)$.

The potential trending lines shown in Figure 9 presented the best fit to the relation between the functional and rheological properties measured at $25^{\circ} \mathrm{C}$. Apparently the relation between 
both properties is not so good at this temperature, since the dispersion of the several points has increased (probably due to the type of rheometer used in this work). Furthermore, the relation between the functional and rheological properties of the different types of binder (BASE, AR and RES) cannot be adjusted by a single trending line, in opposition to the previous relations.

The range of values of the penetration results is visibly inferior to the rheological classification, especially for the RES and AR binders, and thus the rheology axis is presented in a logarithmic scale. Actually, the penetration values of the RES and AR binders show little variation, being always inferior to $50 \mathrm{dmm}$. It is worth to mention that, by analysing the rheology of the different binders for the same penetration value, the RES bitumen is the stiffest material, followed by the BASE bitumen, being the AR binder the softest material (this result is consistent with the best fatigue cracking resistance of this material).

\section{CONCLUSIONS}

The main conclusions that can be drawn from the results of this work are the following:

- during the AR binder production, the interaction of the crumb rubber particles with softer bitumens is clearly superior than with harder bitumens, and thus the morphology of the rubber particles is clearly more influenced by softer bitumens (apparently the rubber particles in contact with softer bitumens swell much more, splitting and releasing a great amount of very small elements recovered in the residual bitumen);

- the functional characterization of binders is more effective for bitumens than for AR modified binders, because the AR functional properties are poorly influenced by the type of base bitumen used (especially at higher temperatures); the AR binder results present greater dispersions, mainly because of the heterogeneity of this material;

- the 150/200 bitumen is rarely used to produce asphalt rubber, but it greatly interacts with crumb rubber, being a very good alternative to produce AR binders with very good characteristics (similar to those obtained with 60/70 bitumens) during the life cycle of the pavement;

- the rheology of AR binders at higher temperatures $\left(160\right.$ to $\left.180^{\circ} \mathrm{C}\right)$ is independent from the type of base bitumen used (trending for the same value of $\mathrm{G}^{*} / \operatorname{sen}(\delta)$ ), although being clearly stiffer than the BASE and RES bitumens at these temperatures (the high stiffness of the $\mathrm{AR}$ binder at those temperatures makes difficult its mixing and application; the increase of temperature beyond $160^{\circ} \mathrm{C}$ almost does not affect the $\mathrm{AR}$ rheological characteristics);

- the lowest values of $\mathrm{G}^{*} / \operatorname{sen}(\delta)$ for AR binders at application temperatures were obtained for lower frequencies (apparently the compaction of AR mixtures should be carried out at low speeds), in opposition to the softening effect of frequency for the BASE and RES bitumens;

- the relation between functional and rheological properties of binders depends on the test temperature, being this relation very good for all the studied binders (BASE, AR and RES) at higher temperatures (dynamic viscosity and R\&B temperature), but not so good at medium operating temperatures (penetration).

\section{ACKNOWLEDGEMENTS}

The materials used in this work were kindly supplied by CEPSA and by RECIPAV to which the authors want to especially thank for their support and for the supply of bitumen and crumb rubber, respectively.

\section{REFERENCES}

Abdelrahman, M.A. \& Carpenter, S.H. 1999. Mechanism of interaction of asphalt cement with crumb rubber modifier. Transportation Research Record 1661: 106-113. 
Airey, G.D., Rahman, M.M. \& Collop, A.C. 2003. Absorption of bitumen into crumb rubber using the basket drainage method. International Journal of Pavement Engineering 4 (2): 105-119.

Artamendi, I., Eastmond, G.C. \& Khalid, H. 2002. Influence of crumb rubber modifier (CRM) from tyre waste on the rheological properties of bituminous binders. Proc. 3rd Int. Conf. Bituminous Mixtures and Pavements, Vol. 1, Thessaloniki, 21-22 November.

Bahia, H.U. \& Davies, R. 1994. Effect of crumb rubber modifiers (CRM) on performance-related properties of asphalt binders. Journal of the Association of Asphalt Paving Technologists 63: 414449.

Bahia, H. \& Davies, R. 1995. Role of Crumb Rubber Content and Type in Changing Critical Properties of Asphalt Binders. Journal of the Association of Asphalt Paving Technologists 64: 130-162.

Billiter, T.C., Chun, J.S., Davison, R.R., Glover, C.J. \& Bullin, J.A. 1997a. Investigation of the curing variables of asphalt-rubber binder. Petroleum Science and Technology 15 (4-5): 445-469.

Billiter, T.C., Chun, J.S., Davison, R.R., Glover, C.J. \& Bullin, J.A. 1996. Investigation of the curing variables of asphalt-rubber binder. American Chemical Society, Division of Fuel Chemistry 41(4).

Billiter, T.C., Davison, R.R., Glover, C.J. \& Bullin, J.A. 1997b. Production of asphalt-rubber binders by high-cure conditions. Transportation Research Record 1586: 50-56.

Gopal, V.T., Sebaaly, P.E. \& Epps, J. 2002. Effect of Crumb Rubber Particle Size and Content on the Low Temperature Rheological Properties of Binders. Transportation Research Board Annual Meeting, Washington D. C., 13-17 January.

Heitzman, M. 1992. State of the Practice-Design and Construction of Asphalt Paving Materials with Crumb Rubber Additive. Report No. FHWA-SA-92-022. Federal Highway Administration.

Navarro, F.J., Partal, P., Martínez-Boza, F. \& Gallegos, C. 2005. Influence of crumb rubber concentration on the rheological behaviour of a crumb rubber modified bitumen. Energy and Fuels 19: 1984-1990.

Rahman, M.M. 2004. Characterisation of Dry Process Crumb Rubber Modified Asphalt Mixtures. Thesis submitted to the University of Nottingham for the degree of Doctor of Philosophy. Nottingham, University of Nottingham.

Read, J., Whiteoak, C.D., 2003. The Shell Bitumen Handbook. Shell Bitumen UK, Thomas Telford Publishing, 5th edition, London.

Singleton, T.M., Airey, G.D. \& Collop, A.C. 2000. Effect of rubber-bitumen interaction on the mechanical durability of impact absorbing asphalt. Proc. 2nd Eurasphalt \& Eurobitume Congress, Vol. 1, Barcelona, 20-22 September.

van de Ven, M. \& Jenkins, K. 2003. Rheological characterization of some (polymer modified) bitumen and bitumen-filler system at compaction and in service temperatures. 6th RILEM Symposium on Performance Testing and Evaluation of Bituminous Materials (PTEBM 2003), Zurich, 14-16 April.

Zanzotto, L. \& Kennepohl, G.J. 1996. Development of rubber and asphalt binders by depolymerization and devulcanization of scrap tires in asphalt. Transportation Research Record 1530: 51-58. 
